\title{
Lateral quantization of spin waves in micron size magnetic wires
}

\author{
C. Mathieu, J. Jorzick, A. Frank, S.O. Demokritov and B. Hillebrands \\ Fachbereich Physik, Universität Kaiserslautern, 67653 Kaiserslautern, Germany
}

\author{
A.N. Slavin \\ B. Bartenlian and C. Chappert \\ IEF, Université Paris Sud, 91405 Orsay, France \\ D. Decanini, F. Rousseaux, and E. Cambril \\ L2M, Bagneux, France
}

Department of Physics, Oakland University, Rochester, Michigan 48309-4401

\begin{abstract}
We report on the observation of quantized surface spin waves in periodic arrays of magnetic $\mathrm{Ni}_{81} \mathrm{Fe}_{19}$ wires by means of Brillouin light scattering spectroscopy. At small wavevectors $\left(\mathrm{q}_{\|} \cong 0-0.9 \cdot 10^{5} \mathrm{~cm}^{-1}\right)$ several discrete, dispersionless modes with a frequency splitting of up to $0.9 \mathrm{GHz}$ were observed for the wavevector oriented perpendicular to the wires. From the frequencies of the modes and the wavevector interval, where each mode is observed, the modes are identified as dipole-exchange surface spin wave modes of the film with quantized wavevector values determined by the boundary conditions at the lateral edges of the wires. With increasing wavevector the separation of the modes becomes smaller, and the frequencies of the discrete modes converge to the dispersion of the dipole-exchange surface mode of a continuous film.
\end{abstract}

Patterned magnetic films are attracting increasing interest due to their potential applications in magnetic storage devices and sensors. Although static properties and coupling phenomena in magnetic films patterned on the micron scale have been studied extensively [1,2,3,4], high-frequency dynamic properties of such films have been rarely investigated. On the other hand, the study of spin wave properties in conventional finite size systems is well established, such as the investigation of so-called Walker-modes in magnetic spheroids [5], and of dipolar-dominated surface modes (Damon-Eshbach modes) in finite-thickness slabs with infinite lateral dimensions [6]. For periodic, micron-sized magnetic structures such a study has been still lacking, likely due to the high requirements both concerning the sample quality and the performance of the Brillouin light scattering experiment to detect the rather weak spin wave signals.

In this Letter we report on the observation of quantization of spin waves in an array of magnetic wires. The quantization effects are identified as being due to the finite width of the wires. The evolution of the Damon-Eshbach mode of a continuous film from the discrete eigenmode spectrum of the wires with increasing wavevector, i.e., with diminishing influence of the finite size effect, is demonstrated and quantitatively described by a model based on quantized dipoleexchange modes. We show that both the frequency values and the wavevector intervals, where these modes are observed, are in a good agreement with our proposed model.

The samples are made of a $200 \AA$ thick permalloy $\left(\mathrm{Ni}_{81} \mathrm{Fe}_{19}\right)$ film deposited in UHV onto a $\mathrm{Si}(111)$ substrate by means of e-beam evaporation. Patterning was performed using X-ray lithography. The patterning masks were fabricated by means of a JEOL 5D2U nanopattern generator at $50 \mathrm{keV}$. $\mathrm{X}$-ray exposure was performed at the super-ACO facility (LURE, Orsay, France) using a negative resist with a lift-off process with $\mathrm{Al}$ coating and ion milling. Two samples with periodic arrays of wires with a wire width $\mathrm{w}=1.8 \mu \mathrm{m}$ and distances between the centers of the wires, $\Lambda$, of $2.5 \mu \mathrm{m}$ and $4 \mu \mathrm{m}$ (i.e., wire separations of $0.7 \mu \mathrm{m}$ and $2.2 \mu \mathrm{m}$ ) were prepared for the investigation of the interaction between the wires. The length $\mathrm{L}$ of the wires was $500 \mu \mathrm{m}$. The patterned area for each of the two investigated samples was $500 \times 500 \mu^{2}$.

The spin wave properties were investigated by using a computer controlled tandem Fabry-Perot interferometer as described elsewhere [7]. Laser light of a single-moded $\mathrm{Ar}^{+}$ laser operating at a wavelength of $\lambda_{\text {Laser }}=514.5 \mathrm{~nm}$ with a power of $50 \mathrm{~mW}$ was focused onto the sample, and the frequency spectrum of the backscattered light was analyzed. The external field of 500 Oe was applied along the wires. The in-plane wavevector $\mathrm{q}_{\|}=\left(4 \pi / \lambda_{\text {Laser }}\right) \cdot \sin \theta$ of the spin waves tested in the experiment was oriented perpendicular to the wires. Its value was varied by changing the angle of light incidence, $\theta$, measured against the surface normal.

Fig. 1 shows the anti-Stokes side of a typical Brillouin light scattering spectrum for a wavevector $\mathrm{q}_{\|}=0.3 \cdot 10^{5} \mathrm{~cm}^{-1}$ of the sample with $0.7 \mu \mathrm{m}$ separation between the wires. Near 7.1, 8.0, 8.8 and $9.6 \mathrm{GHz}$ four distinct modes of magnetic excitations are observed. By varying the magnitude of the wavevector, $\mathrm{q}_{\|}$, the spin wave dispersion is obtained, as displayed in Fig. 2. Shown are the data for the wires with a separation of $2.2 \mu \mathrm{m}$ (top), $0.7 \mu \mathrm{m}$ (middle) and, for reference, for a continuous film (bottom). In the region of low wavevectors the spin wave modes show a disintegration of the continuous dispersion of the Damon-Eshbach mode into several discrete, resonance-like modes with a frequency spacing of approximately $0.9 \mathrm{GHz}$ between the lowest lying modes. There is no significant difference between the data obtained from the wires with $0.7 \mu \mathrm{m}$ and $2.2 \mu \mathrm{m}$ separation, 
indicating that the mode splitting is purely caused by the quantization of the spin waves due to the finite wire width, and the magneto-dipole interaction between wires is small for both studied separations.

Studying the experimentally observed spin wave dispersion of the patterned structures in more detail we note the following: (i) For low wavevector values $\left(\mathrm{q}_{\|} \cong 0-0.9 \cdot 10^{5} \mathrm{~cm}^{-1}\right)$ the discrete modes do not show any noticeable dispersion and they behave like standing wave resonances. (ii) The lowest mode appears at zero wavevector, whereas all higher modes appear at nonzero wavevector and the value of this lower "cut-off" wavevector increases with the mode number. (iii) There is a transition region $\left(\mathrm{q}_{\|} \cong 1-1.3 \cdot 10^{5} \mathrm{~cm}^{-1}\right)$ between the well resolved dispersionless modes, and the continuous film-like dispersion, where are discrete modes, but the mode separation is close or slightly below the experimental frequency resolution in the BLS experiment $(\approx 0.1 \mathrm{GHz})$. (iv) For large values of the wavevector $\left(\mathrm{q}_{\|}>1.3 \cdot 10^{5} \mathrm{~cm}^{-1}\right)$ the dispersion of the patterned film converges to the dispersion of a continuous film.

It is worth noting, that an early indication of the existence of discrete modes in the spin wave spectrum of an array of permalloy wires was reported by Gurney et al. [8]. Three discrete spin wave modes were observed at a single value of the in-plane wavevector $\mathrm{q}_{\|}$of $1.2 \cdot 10^{5} \mathrm{~cm}^{-1}$. The authors were not able to identify the nature of the modes.

In an infinite ferromagnetic film of thickness $d$ with the direction of magnetization aligned perpendicular to the spin-wave wavevector and within the film plane, the dipoleexchange spin wave spectrum of the film consists of the dipole-dominated (Damon-Eshbach) mode and the higher frequency exchange-dominated modes with large perpendicular wavevector components, $\mathrm{q}_{\perp}$, taking the quantized values of $m \pi / d$ with $m=1,2,3, \ldots .[9,10]$. Although these exchange-dominated modes are also observed in our experiments, they are not further considered here since their frequencies, which are much higher than the frequencies of the discrete modes, depend only on the film thickness (and not on the in-plane pattern parameters).

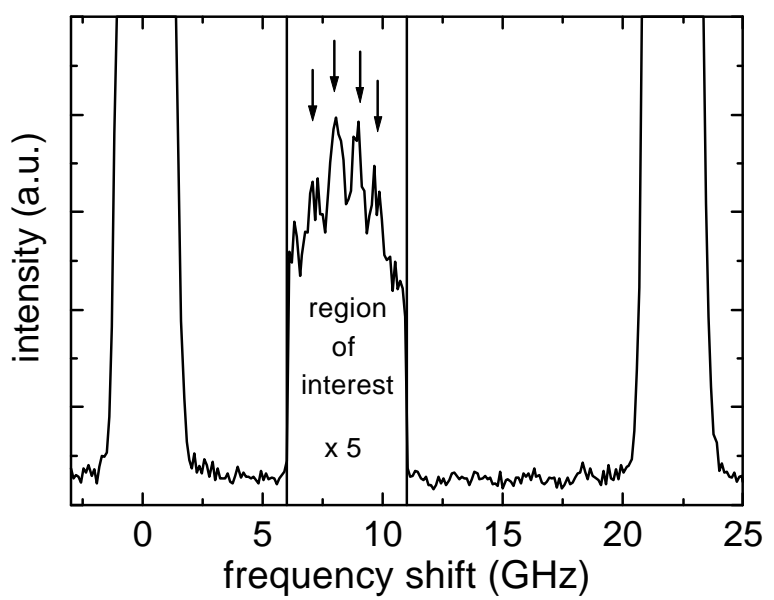

Fig. 1: Brillouin light scattering spectrum of the sample with a wire width of $1.8 \mu \mathrm{m}$ and a wire separation of $0.7 \mu \mathrm{m}$, demonstrating the existence of several discrete spin wave modes with a mode splitting of about $0.9 \mathrm{GHz}$ (indicated by arrows). The applied field is $0.5 \mathrm{kOe}$. The in-plane wavevector $\mathrm{q}_{\|}$is $0.3 \cdot 10^{5}$ $\mathrm{cm}^{-1}$.
In the following we assume a cartesian coordinate system oriented such that the normal of the pattern area points into the $\mathrm{x}$-direction, the wavevector $\mathrm{q}_{\|}$points into the $\mathrm{y}$-direction and the wire axes and the magnetization are aligned parallel to the z-axis.

In a magnetic wire of a finite width $\mathrm{w}$ the spin wave spectrum is modified by the width-dependent quantization of the wavevector. If $w$ is much larger than the height $d$ the usual approximate method is to introduce the fictitious boundary conditions for the magnetization $\mathrm{m}$ at the film's lateral edges [11]:

$$
\frac{\partial m}{\partial y}+\left.D m\right|_{y= \pm w / 2}=0 \text {. }
$$

Since the "pinning" parameter D, determined by the effective surface anisotropy at the lateral edges of the wire, is not known, we shall use it below as a fitting parameter. We also assume that the magnetization distribution of the lateral discrete modes has the form

$$
\begin{aligned}
& m_{n}(y)=A_{n} \cos \left(q_{n}(y+w / 2)\right)+B_{n} \sin \left(q_{n}(y+w / 2)\right), \\
& -w / 2<y<w / 2 .
\end{aligned}
$$

Substituting Eq. (2) for $m$ into the boundary conditions Eq. (1), we obtain a relation between the coefficients $B_{n}$ and $A_{n}$ of the form

$$
B_{n}=A_{n} \frac{D}{q_{n}}
$$

and the transcendental equation determining the discrete values of the lateral wavevector $\mathrm{q}_{\mathrm{n}}[12]$ :

$$
\tan \left(q_{n} w\right)=\frac{2 q_{n} D}{q_{n}^{2}-D^{2}} .
$$
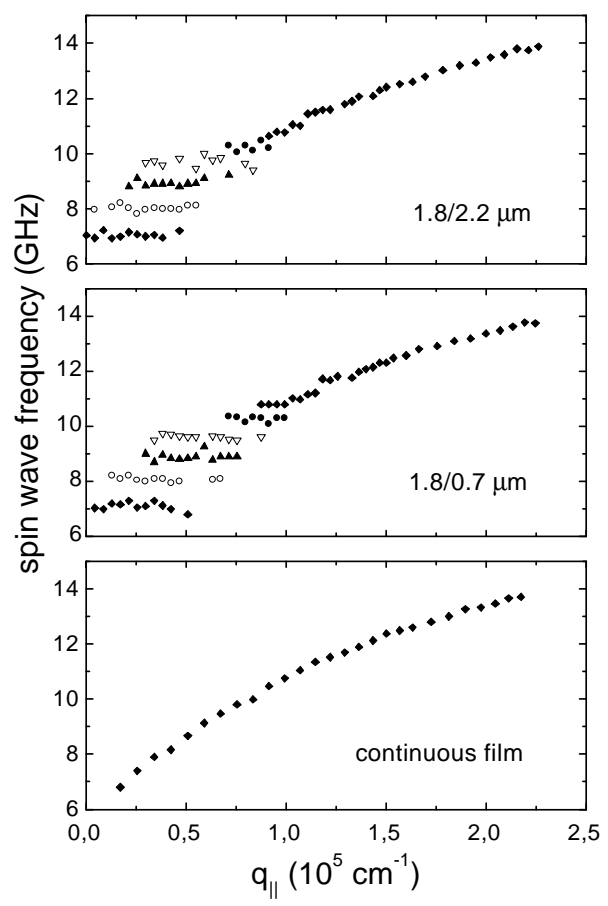

Fig. 2: Obtained spin wave dispersion curves for an array of wires of $1.8 \mu \mathrm{m}$ width and a separation of the wires of $2.2 \mu \mathrm{m}$ (top) and $0.7 \mu \mathrm{m}$ (middle). The bottom panel shows a spin wave spectrum of a continuous film for reference. The thickness of the wires and the continuous film is $200 \AA$, the external field applied along the wire axes is $0.5 \mathrm{kOe}$. 
The frequencies of the discrete modes for $\mathrm{n}=0,1,2, \ldots$ can be calculated by substituting solutions of Eq. (4) for the discrete wavenumber values, $\mathrm{q}_{\mathrm{n}}$, in the equation of a dipoledominated surface (quasi-Damon-Eshbach) mode of a tangentially magnetized infinite film $[9,10]$. This calculation done for our experimental parameters $(d=200 \AA$, $\mathrm{w}=1.8 \mu \mathrm{m}$, saturation magnetization $4 \pi \mathrm{M}_{\mathrm{s}}=10.2 \mathrm{kG}$, external magnetic field $\mathrm{H}=0.5 \mathrm{kOe}$, exchange constant $\mathrm{A}=10^{-6}$ $\mathrm{erg} / \mathrm{cm}$, gyromagnetic ratio $\gamma / 2 \pi=2.95 \mathrm{GHz} / \mathrm{kOe}$ ) and the lateral "pinning" parameter $\mathrm{D}$ chosen to be $\mathrm{D}=2.7 \cdot 10^{3} \mathrm{~cm}^{-1}$ yields values of the mode frequencies of 7.1, 7.9, 8.8, 9.5, 10.2 and $10.8 \mathrm{GHz}$ for the $\mathrm{n}=0 \ldots .5$ modes, which are in good quantitative agreement with the experimentally observed frequency positions of the discrete resonance-like modes shown in Fig. 2. It should be pointed out that the only adjusted parameter in this fit is the pinning parameter, D. With increasing mode number $\mathrm{n}$ the mode separation $\Delta f=f_{\mathrm{n}+1}-f_{\mathrm{n}}$ becomes smaller than the frequency resolution in the BLS experiment, and the observed mode frequencies are well described by the dispersion equation for a film of infinite width and continuous values of $\mathrm{q}_{\|}$.
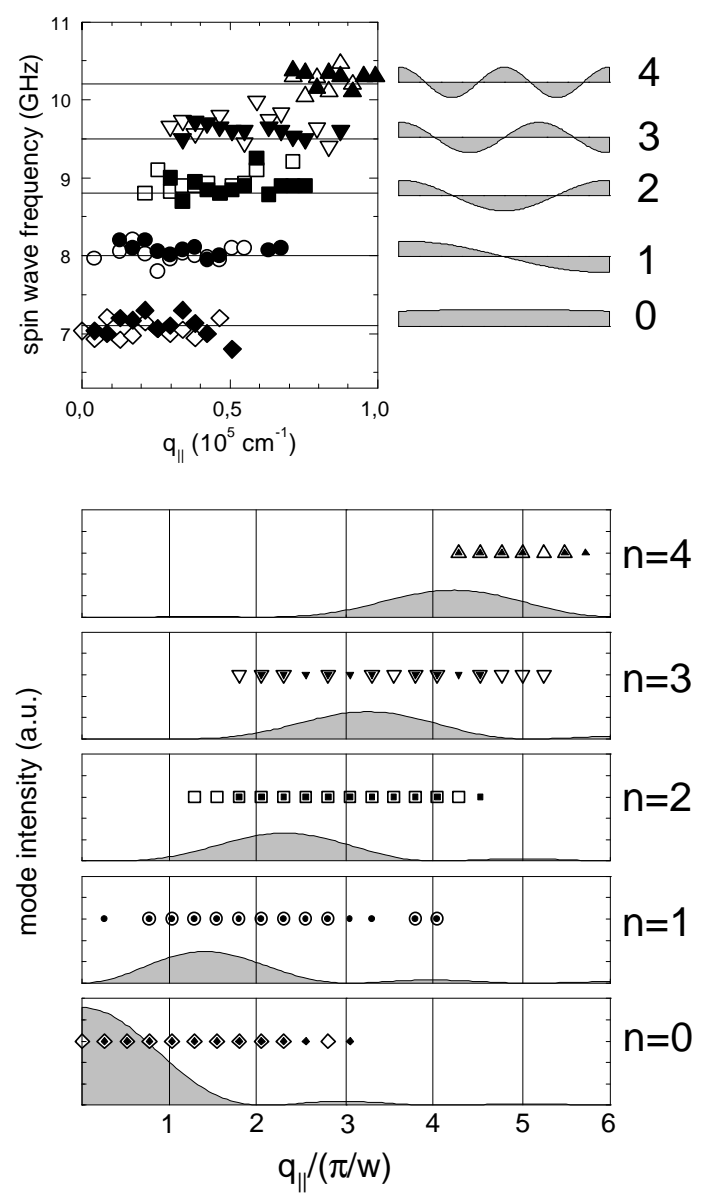

Fig. 3:Top/Left: Comparison of the calculated frequencies of the five lowest quantized dipole-exchange surface spin wave modes, $n=0-4$, (horizontal lines) with the experimental results for the arrays of wires with $0.7 \mu \mathrm{m}$ (full symbols) and $2.2 \mu \mathrm{m}$ (open symbols) wire separation. Top/Right: Calculated magnetization profiles of these modes $(n=0-4)$ as described in the text. Bottom: Mode Fourier-intensity as a function of $\mathrm{q}_{\|}$in units of $\pi / w$ for these modes $(n=0-4)$. The up and down triangles denote the values of $\mathrm{q}_{\|}$for the samples with $0.7 \mu \mathrm{m}$ and $2.2 \mu \mathrm{m}$ separation, respectively, where spin wave excitations are detected.
The comparison of the theoretically calculated frequencies for the modes with $n=0-4$ with our experimentally measured values is shown in the upper left part of Fig. 3. The full and open symbols denote the values of the samples with $0.7 \mu \mathrm{m}$ and $2.2 \mu \mathrm{m}$ wire separation, respectively. The magnetization profiles (see cf. Eq. (2)) along the wire width w of these modes are depicted in the upper right part of Fig. 3. We would like to note, that the value of the fitted "pinning" parameter D affects mostly the frequency position and the magnetization profile of the lowest mode $(\mathrm{n}=0$, $\left.q_{0}=5.26 \cdot 10^{3} \mathrm{~cm}^{-1}\right)$. For higher modes $(\mathrm{n}>0) \mathrm{q}_{\mathrm{n}}$ is much larger than D and the solutions of Eq. (4) are close to $q_{n}=n \pi / w$, only weakly dependent on the value of $\mathrm{D}$. Note here that the obtained value of $\mathrm{D}$ corresponds to the very small surface anisotropy constant $\mathrm{K}_{\mathrm{S}}=2.7 \cdot 10^{-3}$ $\mathrm{erg} / \mathrm{cm}^{2}$.

Above interpretation of the observed discrete modes is further supported by the data of the wavevector intervals in which the modes can be observed. By changing the angle of the incident light a wide range of spin wave wavevectors can be accurately investigated. If infinite traveling waves are under investigation, only one wave with $m(y) \propto \exp \left(i q_{\|} y-i \omega t\right)$ will contribute to the inelastic light

scattering process at a definite incident angle of light, $\theta$, which determines the wavevector $\mathrm{q}_{\|}$. If the investigated spin wave mode is described by an arbitrary mode profile $\mathrm{m}(\mathrm{y})$, the light scattering process for such a mode takes place within a certain interval of $\theta$ or $\mathrm{q}_{\|}$and the scattering intensity is determined by the Fourier component $\mathrm{m}\left(\mathrm{q}_{\|}\right)$of the mode: $I \propto\left|m\left(\mathrm{q}_{\|}\right)\right|^{2}$. For a spin wave mode existing in the wire of width $w, m(y)$ is zero outside the wire, and the Fourier component $\mathrm{m}\left(\mathrm{q}_{\|}\right)$is given by:

$$
m\left(q_{\|}\right)=\int_{-w / 2}^{w / 2} m(y) \exp \left(i q_{\|} y\right) d y .
$$

Using Eq. (5), the Fourier components $\left|\mathrm{m}_{\mathrm{n}}\left(\mathrm{q}_{\|}\right)\right|$for the discrete modes (see Eq. (2)) are calculated. The resulting intensity profiles $\mid \mathrm{m}_{\mathrm{n}}(\mathrm{q} \|)^{2}$ of the lowest five modes are shown in Fig. 3 (bottom) for the $\mathrm{q}_{\|}$-range between 0 and $1 \cdot 10^{5} \mathrm{~cm}^{-1}(\approx 6 \pi / \mathrm{w})$. These calculated intensity profiles should clearly define the intervals of $\mathrm{q}_{\|}$, in which the corresponding spin wave modes should be detectable. For comparison, full and open symbols in Fig. 3 (bottom) denote the values of $\mathrm{q}_{\|}$, at which each mode was observed for the samples with wire separations of 0.7 and $2.2 \mu \mathrm{m}$, respectively. There is a good agreement between the results of this calculation and the experimentally measured wavevector intervals, where the corresponding discrete modes are observed. In summary we have clearly observed a spin wave mode quantization effect in a periodic array of magnetic wires. The observed discrete modes can be interpreted as resulting from the width-dependent quantization of the dipoleexchange surface spin wave mode (quasi-Damon-Eshbach mode) of an infinite film. Both the frequency positions and the wavevector intervals, where the discrete modes are observed, support our interpretation. For larger wavevectors a 
quasi-continuous spin wave dispersion resembling closely the dipole-exchange dispersion of a continuous film is obtained. No indication for a zone folding effect due to the periodic arrangement of the wires was found.

Support by the Deutsche Forschungsgemeinschaft and the National Science Foundation of the U.S.A. (Grant \# DMR 9701640 ) is gratefully acknowledged.

\section{References:}

1 J.F. Smyth, S. Schultz, D.R. Fredkin, D.P. Kern, S.A. Rishton, H. Schmid, M. Cali, and T.R. Koehler, J. Appl. Phys. 69, 5262 (1991).

2 A. Maeda, M. Kume, T. Ogura, K. Kukori, T. Yamada, M. Nishikawa, and Y. Harada, J. Appl. Phys. 76, 6667 (1994).

3 B. Hillebrands, C. Mathieu, M. Bauer, S.O. Demokritov, B. Bartenlian, C. Chappert, D. Decanini, F. Rousseaux, and F. Carcenac, J. Appl. Phys. 81, 4993 (1997).

4 C. Mathieu, C. Hartmann, M. Bauer, O. Büttner, S. Riedling, B. Roos, S.O. Demokritov, and B. Hillebrands, Appl. Phys. Lett. 70, 2912 (1997).

5 L.R. Walker, Phys. Rev. 105, 390 (1957); R.L. White and J.H. Solt, Phys. Rev. 104, 56 (1956).

6 R.W. Damon and J.R. Eshbach, J. Phys. Chem. Solids 19, 308 (1961).

7 R. Mock, B. Hillebrands, and J.R. Sandercock, J. Phys. E 20, 656 (1987).

8 B.A. Gurney, P. Baumgart, V. Speriosu, R. Fontana, A. Patlac, T. Logan, and P. Humbert, Digest of the International Conference on Magnetic Films and Surfaces, P7.12, p.474, Glasgow (1991).

9 R.E. De Wames and T. Wolfram, J. Appl. Phys. 41, 987 (1970).

10 B. Hillebrands, Phys. Rev. B 41, 530 (1990).

11 A.G. Gurevich and G.A. Melkov, Magnetization Oscillations and Waves (CRC Press, New York, 1996).

12 R.F. Soohoo, Magnetic Thin Films, Harper \& Row, New York, 1964, p. 231. 